\title{
SCATTERING PHASE ASYMPTOTICS WITH FRACTAL REMAINDERS
}

\author{
SEMYON DYATLOV AND COLIN GUILLARMOU
}

\begin{abstract}
For a Riemannian manifold $(M, g)$ which is isometric to the Euclidean space outside of a compact set, and whose trapped set has Liouville measure zero, we prove Weyl type asymptotics for the scattering phase with remainder depending on the classical escape rate and the maximal expansion rate. For Axiom A geodesic flows, this gives a polynomial improvement over the known remainders. We also show that the remainder can be bounded above by the number of resonances in some neighbourhoods of the real axis, and provide similar asymptotics for hyperbolic quotients using the Selberg zeta function.
\end{abstract}

In this paper, we derive high energy asymptotics for the spectral shift function, or the scattering phase, with remainders which depend on the dynamic behavior of the underlying classical system. Our main setting is an $n+1$ dimensional Riemannian manifold $(M, g)$ which is Euclidean near infinity, but we shall also consider in the second part of the paper the case of convex co-compact hyperbolic quotients $\Gamma \backslash \mathbb{H}^{n+1}$ using a different approach. If $\Delta$ is the (nonnegative) Laplace-Beltrami operator on $M$ and $\Delta_{0}$ is a reference operator (such as the Laplacian on the free space when $(M, g)$ is a metric perturbation of the Euclidean space), then the spectral shift function is the trace of the difference between the spectral projectors of $\Delta$ and $\Delta_{0}$ - see (1.1) and (1.2). The scattering phase is the phase of the determinant of the relative scattering matrix. In a very general setting, these two functions are equal almost everywhere on the absolutely continuous spectrum, as shown in the classical work of Birman-Krein [BiKr], thus we will denote both of them by the symbol $s(z)$, where $z \in \mathbb{R}$ is the frequency.

Our recent work [DyGu] contains a local Weyl law with a remainder expressed in terms of classical escape rate - in particular, if the metric has constant curvature -1 near the trapped set $K$ and the Hausdorff dimension of $K$ is $\operatorname{dim}_{H}(K)=2 \delta+1$, then the remainder is $\mathcal{O}\left(z^{\delta+}\right)$. If $M$ is Euclidean near infinity, we use the commutator method of Robert [Ro] to deduce an asymptotic expansion of $s(z)$ with the same remainder - see Theorem 1 . This seems to be the first result on spectral asymptotics with a fractal remainder which is intermediate between the usual Weyl law and the non-trapping case where there is a complete expansion at high frequency. By contrast, the best known remainder for Weyl law on compact manifolds with chaotic geodesic flows is $\mathcal{O}\left(z^{n} / \log z\right)$, see Bérard [Bé]. 
We next give several other estimates on the spectral shift function in different settings, with remainders related to the one discussed in the previous paragraph. First of all, the function $s(z)$ admits a meromorphic extension into the whole complex plane, and the asymptotic behavior of $s(z)$ for large real $z$ is related to the distribution of its poles, known as scattering poles or resonances. In the Euclidean near infinity setting, we get an asymptotic expansion of the scattering phase with remainder depending on the number of resonances in the ball of size $c \log (z)$ centered at $z$, for some $c$ depending on the injectivity radius - see Theorem 2. Next, for the case of hyperbolic surfaces $\Gamma \backslash \mathbb{H}^{2}$, we get an asymptotic expansion of $s(z)$ using the Selberg zeta function - see Theorem 3.

1.1. Euclidean near infinity case - an application of [DyGu]. Let $g_{0}$ and $g_{1}$ be two smooth metrics on a manifold $M$. When there exists a compact set $N \subset M$ such that each $\left(M \backslash N, g_{j}\right)$ is isometric to $\mathbb{R}^{n+1} \backslash B\left(0, R_{0}\right)$ for some $R_{0}>0$, we define (up to a constant) the spectral shift function $s(z)$ for the pair $\left(\Delta_{g_{1}}, \Delta_{g_{0}}\right)$ by duality: for all $\varphi \in C_{0}^{\infty}(\mathbb{R})$,

$$
\int_{0}^{\infty} \varphi(z) \partial_{z} s(z) d z=\operatorname{Tr}\left(\varphi\left(\sqrt{\Delta_{g_{0}}}\right)-\varphi\left(\sqrt{\Delta_{g_{1}}}\right)\right)
$$

where it is straightforward to see that the trace is well defined, using that $g_{0}=g_{1}$ outside a compact set. In this setting, $s(z)$ will be a smooth function on $(0, \infty)$ and it is equal, up to a multiplicative constant, to the phase of the relative scattering operator by the work of Birman-Krein [BiKr].

We shall also be interested in the case where $\left(M_{0}, g_{0}\right)$ and $\left(M_{1}, g_{1}\right)$ are two Riemannian manifolds such that for each $j=0,1$ there is a compact set $N_{j} \subset M_{j}$ with $M_{j} \backslash N_{j}$ isometric to $\mathbb{R}^{n+1} \backslash B\left(0, R_{0}\right)$. The spectral shift function for the pair $\left(\Delta_{g_{1}}, \Delta_{g_{0}}\right)$ can then be defined using the black-box trace [Sj97] by the formula

$$
\int_{0}^{\infty} \varphi(z) \partial_{z} s(z) d z=\operatorname{Tr}_{\mathrm{bb}}\left(\varphi\left(\sqrt{\Delta_{g_{0}}}\right)-\varphi\left(\sqrt{\Delta_{g_{1}}}\right)\right)
$$

where the trace $\operatorname{Tr}_{\mathrm{bb}}$ is defined in (2.1).

Before we state our result, we need to define a few geometric quantities. Let $(M, g)$ be a Riemannian manifold such that, for some compact set $N \subset M$, the end $(M \backslash N, g)$ is isometric to $\mathbb{R}^{n+1} \backslash B\left(0, R_{0}\right)$ for some $R_{0}>0$. Let $g^{t}$ be the geodesic flow acting on the unit cotangent bundle $S^{*} M$. The trapped set $K \subset S^{*} M$ is defined as follows: $(m, \nu)$ lies in $K$ if and only if the corresponding geodesic $g^{t}(m, \nu)$ lies entirely in some compact subset of $S^{*} M$. Denote by $\mu_{L}$ the Liouville measure on $S^{*} M$ generated by the function $\sqrt{p(m, \nu)}=|\nu|_{g}$. The set $\mathcal{T}(t)$ of geodesics trapped for time $t>0$ is defined as

$$
\mathcal{T}(t):=\left\{(m, \nu) \in S^{*} M \mid m \in N, \pi\left(g^{t}(m, \nu)\right) \in N\right\}
$$


where $\pi: S^{*} M \rightarrow M$ is the canonical projection. We define the maximal expansion rate of the geodesic flow as follows:

$$
\Lambda_{\max }:=\limsup _{|t| \rightarrow+\infty} \frac{1}{|t|} \log \sup _{(m, \nu) \in \mathcal{T}(t)}\left\|d g^{t}(m, \nu)\right\| .
$$

The norm on the right hand side is with respect to the Sasaki metric. Using our recent work [DyGu], we obtain

Theorem 1. Let $(M, g)$ be a smooth Riemannian manifold for which there is a compact subset $N$ such that $M \backslash N$ is isometric to $\mathbb{R}^{n+1} \backslash B\left(0, R_{0}\right)$. Assume that the trapped set $K$ has Liouville measure 0 and let $s(z)$ be the spectral shift function associated to the pair $\left(\Delta_{g}, \Delta_{\mathbb{R}^{n+1}}\right)$ defined by (1.2). Let $\Lambda_{\max }$ be defined in (1.4) and $\Lambda_{0}>\Lambda_{\max }$, then there exist some coefficients $c_{j}$ such that as $z \rightarrow \infty$ we have for all $L \in \mathbb{N}$

$$
s(z)=\sum_{j=0}^{L} c_{j} z^{n+1-j}+\mathcal{O}\left(z^{n} \mu_{L}\left(\mathcal{T}\left(\Lambda_{0}^{-1} \log |z|\right)\right)\right)+\mathcal{O}\left(|z|^{n-L}\right) .
$$

The coefficients $c_{j}$ are integrals of local Riemannian invariants, they appear in the small time asymptotics for the local trace of the heat kernel or Schrödinger propagator (e.g. see Robert [Ro]); for instance, $c_{0}=C_{n}\left(\operatorname{Vol}_{\text {eucl }}\left(B\left(0, R_{0}\right)\right)-\operatorname{Vol}_{g}(N)\right)$ where $C_{n}$ is a universal constant depending only on $n$. When $K=\emptyset$, we recover a full expansion for $s(z)$, when $K \neq \emptyset$, the remainder is not better than $\mathcal{O}(1)$.

In the proof, we need to assume that $M$ is Euclidean at infinity for (1.5) since we use the commutator method of Robert [Ro] to express the scattering phase as a trace of the spectral projector in a compact region, and then we apply our estimate [DyGu, Theorem 3] on the expansion as $h \rightarrow 0$ of the trace $\operatorname{Tr}\left(A \mathbb{1}_{[0, \lambda]}\left(h^{2} \Delta_{g}\right)\right)$ when $\lambda>0$ is in a compact interval and $A \in \Psi^{0}(M)$ is any compactly supported semiclassical pseudodifferential operator. It is likely that the result extends to perturbations of $\mathbb{R}^{n+1}$ which are not compactly supported.

We can now specialize to particular cases where the flow is uniformly partially hyperbolic in the following sense: there exists $\lambda>0$ and an invariant splitting of $T S^{*} M$ over $K$ into continuous subbundles

$$
T_{z} S^{*} M=E_{z}^{c s} \oplus E_{z}^{u}, \quad \forall z \in K
$$

such that the dimensions of $E^{u}$ and $E^{c s}$ are constant on $K$ and for all $\varepsilon>0$, there is $t_{0} \in \mathbb{R}$ such that

$$
\forall z \in K, \forall t \geq t_{0},\left\{\begin{array}{l}
\forall v \in E_{z}^{u},\left\|d g_{z}^{t} v\right\| \geq e^{\lambda t}\|v\| \\
\forall v \in E_{z}^{c s},\left\|d g_{z}^{t} v\right\| \leq e^{\varepsilon t}\|v\| .
\end{array}\right.
$$

We can define $J^{u}$, the unstable Jacobian of the flow, by

$$
J^{u}(z):=-\left.\partial_{t}\left(\left.\operatorname{det} d g^{t}(z)\right|_{E_{z}^{u}}\right)\right|_{t=0}
$$


where $d g^{t}: E_{z}^{u} \rightarrow E_{g^{t}(z)}^{u}$ and the determinant is defined using the Sasaki metric (to choose orthonormal bases in $E^{u}$ ). The topological pressure of a continuous function $\varphi: K \rightarrow \mathbb{R}$ with respect to the flow can be defined by the variational formula

$$
P(\varphi):=\sup _{\mu \in \mathcal{M}(K)}\left(h_{\mu}\left(g^{1}\right)+\int \varphi d \mu\right)
$$

where $\mathcal{M}(K)$ is the set of $g^{t}$-invariant Borel probability measures and $h_{\mu}\left(g^{1}\right)$ is the measure theoretic entropy of the flow at time 1 with respect to $\mu$. Young [Yo] proved that for uniformly partially hyperbolic flows, the classical escape rate $\lim _{t \rightarrow+\infty} \frac{1}{t} \log \left(\mu_{L}(\mathcal{T}(t))\right.$ is equal to $P\left(J^{u}\right)$. Combining this with Theorem 1, we obtain

Corollary 1.1. Let $(M, g)$ be a Riemannian manifold satisfying the assumptions of Theorem 1, and $\Lambda_{0}>\Lambda_{\max }$. If the geodesic flow is uniformly partially hyperbolic on $K \neq \emptyset$ with negative topological pressure $P\left(J^{u}\right)<0$, then

$$
s(z)=\sum_{j=0}^{n+1} c_{j} z^{n+1-j}+\mathcal{O}\left(z^{n+P\left(J^{u}\right) / \Lambda_{0}}\right) .
$$

In particular, if $g$ has curvature -1 near the trapped set $K$ and the latter has Hausdorff dimension $2 \delta+1$, then

$$
s(z)=\sum_{j=0}^{[n+1-\delta]} c_{j} z^{n+1-j}+\mathcal{O}\left(z^{\delta+}\right) .
$$

When the flow is Axiom A, one has $P\left(J^{u}\right)<0$ by Bowen-Ruelle [BoRu, Theorem 5]. We refer the reader to [DyGu, Appendix B] for more discussions about the classical escape rate and the Hausdorff dimension of the trapped set.

1.2. Relation with resonances. We also compare these asymptotics to the counting function of resonances for the Laplacian $\Delta_{g}$ in regions close to the continuous spectrum. By definition, resonances are poles of the meromorphic continuation of the resolvent $R(z)=\left(\Delta_{g}-z^{2}\right)^{-1}$ from $\operatorname{Im}(z)<0$ to $z \in \mathbb{C}$ as an operator mapping $L_{\text {comp }}^{2}(M)$ to $L_{\text {loc }}^{2}(M)$. We show

Theorem 2. Let $(M, g)$ be a smooth Riemannian manifold which is isometric to $\mathbb{R}^{n+1} \backslash$ $B\left(0, R_{0}\right)$ outside a compact set $N$, with $n+1$ odd, and let $\mathcal{R}$ be the set of resonances of $\Delta_{g}$ in $\{\operatorname{Im}(z) \geq 0\}$. If $t_{0}$ is the radius of injectivity of $g$, and if for some $c>0$ satisfying $\mathrm{ct}_{0}>n+1$ the following estimate holds for $z>0$ large

$$
\sharp\{\rho \in \mathcal{R} ;|z-\rho| \leq c \log (z)\}=\mathcal{O}\left(z^{\alpha}\right),
$$

for some $\alpha \in[0, n]$, then the spectral shift function defined by (1.2) satisfies the asymptotics as $z \rightarrow \infty$

$$
s(z)=\sum_{j=0}^{n+1} c_{j} z^{n+1-j}+\mathcal{O}\left(z^{\alpha}\right) .
$$


The proof uses some ideas of Melrose [Me88] by expressing the derivative of $s(z)$ in terms of resonances through a Poisson formula. Dimassi [Di] showed that $s(z)$ has a full expansion if there are no resonances in a logarithmic neighbourhood of the real axis; our result is (in a way) a generalization of [Di].

Sjöstrand-Zworski [SjZw07] proved that if the geodesic flow is hyperbolic on $K$, then

$$
\sharp\{\rho \in \mathcal{R} ;|z-\rho| \leq c\}=\mathcal{O}\left(z^{m}\right)
$$

for all $m$ satisfying $2 m+1>\operatorname{dim}_{M}(K)$, where $\operatorname{dim}_{M}$ denotes the Minkowski dimension (in case of pure dimensions, $m$ can be taken to be $\left.\left(\operatorname{dim}_{M}(K)-1\right) / 2\right)$. For semiclassical operators with analytic coefficients satisfying hyperbolicity assumptions, Sjöstrand [Sj90, Theorem 4.6] gives estimates for numbers of resonances in boxes of real part of size $z^{1 / 2}$ and imaginary part of any size between 1 and $o(z)$; it is feasible that in combination with the second microlocalization analysis of [SjZw07] to handle the real parts, this method gives in particular the estimate (1.9) with $\alpha$ any number such that $2 \alpha+1>\operatorname{dim}_{M}(K)$. It is not clear whether an estimate of the type (1.10) for resonances in the logarithmic regions (1.9) holds for general $C^{\infty}$ metrics.

1.3. Hyperbolic quotients. In the hyperbolic near infinity setting, there is no natural model operator to define a spectral shift function, however in even dimension one can use the construction of the second author [Gu]. In dimension 2, GuillopéZworski $[\mathrm{GuZw}$ ] defined also a relative scattering phase $s(z)$ by using the Laplacian on the funnels with Dirichlet condition on a curve separating the funnels and a compact region of $M$. In constant curvature $\Gamma \backslash \mathbb{H}^{2}$, this curve can be taken to be the boundary of the convex core, made of disjoint closed geodesics, and $s(z)$ is related to the argument of the Selberg zeta function $Z_{\Gamma}(1 / 2+i z)$ of $\Gamma$ for $z>0$. Using $Z_{\Gamma}(\lambda)$, the estimate on its growth in strips proved by Guillopé-Lin-Zworski [GuLiZw], and some classical results of holomorphic functions, we can compare our microlocal result to see that, at least without more dynamical assumptions, our estimate seems almost sharp:

Theorem 3. Let $M=\Gamma \backslash \mathbb{H}^{2}$ be a convex co-compact hyperbolic surface and let $s(z)$ be the scattering phase defined in [GuZw] using the Laplacian on the funnels with Dirichlet condition at the boundary of the convex core. Let $\delta \in(0,1)$ be the Hausdorff dimension of the limit set of $\Gamma$, or equivalently $2 \delta+1$ is the Hausdorff dimension of the trapped set $K$. Then:

1) $s(z)$ satisfies the asymptotics as $z \rightarrow+\infty$

$$
s(z)=\frac{1}{4 \pi} \operatorname{Vol}(N) z^{2}+R(z), \quad|R(z)|=\mathcal{O}\left(z^{\delta}\right), \quad\left|\int_{0}^{z} R(t) d t\right|=\mathcal{O}\left(z^{\delta}\right)
$$


where $N$ is the convex core of $M$.

2) the Breit-Wigner formula holds: for all $z \in[T-\sigma, T+\sigma]$ with $T$ large and $\sigma$ fixed

$$
\partial_{z} s(z)=\frac{1}{4 \pi} \operatorname{Vol}(N) z^{2}+\frac{1}{\pi} \sum_{s \in \mathcal{R}_{\sigma+1}} \frac{\operatorname{Im}(\rho)}{|z-\rho|^{2}}+\mathcal{O}\left(T^{\delta}\right)
$$

where $\mathcal{R}_{\sigma+1}$ denotes the set of resonances in the disc of radius $\sigma+1$ centered at $T$.

3) If $\Gamma$ is an infinite index sugroup of an arithmetic group derived from a quaternion algebra, and if $\delta>3 / 4$, then for all $\varepsilon>0, R(z)$ is not an $\mathcal{O}\left(z^{2 \delta-3 / 2-\varepsilon}\right)$.

The statement 3) is essentially restating a result proved by Jakobson-Naud [JaNa]. A result similar to Theorem 3 (except statement 3)) is also true for even dimensional convex co-compact hyperbolic Schottky manifolds, see Theorem 5 in Section 3.2.

When $\delta<1 / 2$ in dimension 2, there is actually an exact formula for $s(z)$ as a converging infinite sum over closed geodesics, see (3.7).

1.4. Previous results. The asymptotics for the scattering phase has a long history, we will only give references related to the settings we use in this paper. The first result giving an asymptotics with the leading term was announced in Buslaev [Bu] for a perturbation of $\mathbb{R}^{d}$ by a non-trapping obstacle, this was shown with non-sharp remainder in Majda-Ralston [MaRa1] for the strictly convex case, and by JensenKato [JeKa] for star-shaped domains; an asymptotics with remainder $\mathcal{O}\left(z^{d-3}\right)$ was proved for non-trapping compact metric perturbations in Majda-Ralston [MaRa2]. Then, Petkov-Popov [PePo] gave a full asymptotic expansion in powers of $z$ for nontrapping obstacle perturbations of $\mathbb{R}^{d}$ and a sharp estimate with $\mathcal{O}\left(z^{d-1}\right)$ remainder was shown by Melrose [Me88] for general obstacles $\Omega$ in odd dimensions using the counting function of resonances in large disks of $\mathbb{C}$. When the set of transversally reflected geodesics is of measure 0 , [Me88] obtained a second term of the form $\operatorname{Vol}(\partial \Omega) z^{d-1}$ with remainder $o\left(z^{d-1}\right)$ using a Duistermaat-Guillemin/Ivrii type argument.

Robert [Ro] proved a very general result for (not necessarily compactly supported) second order perturbations of the Euclidean Laplacian on $\mathbb{R}^{d}$ with a remainder $\mathcal{O}\left(z^{d-1}\right)$ and with a complete expansion in powers of $t$ for non-trapping perturbations. In the black-box compact perturbation setting, Christiansen [Chr] gave an estimate with a sharp remainder. In the semiclassical setting, the work of Bruneau-Petkov [BrPe] extends the results of [Ro, Chr]. The Breit-Wigner formula relating resonances and scattering phase in the semi-classical setting appears in particular in $[\mathrm{BrPe}]$ and in Petkov-Zworski [PeZw]. In the setting of hyperbolic surfaces with funnels, GuillopéZworski $[\mathrm{GuZw}$ ] proved a Weyl asymptotics with remainder $\mathcal{O}(z)$ (in fact they dealt with $n_{c}$ cusps as well, in which case there is a second term $-\frac{n_{c}}{\pi} z \log (z)$ before $\mathcal{O}(z)$ ). For convex co-compact hyperbolic manifolds $\Gamma \backslash \mathbb{H}^{n+1}$, the second author [Gu] gave an 
asymptotics with remainder $\mathcal{O}\left(z^{n}\right)$ for the Krein spectral function, and Borthwick [Bo] extended it to compact perturbations.

\section{Asymptotics of the SPECTRAL Shift FUnCtion FOR COMPACT PERTURBATIONS OF THE EUCLIDEAN LAPLACIAN}

2.1. Asymptotics using the trace estimate of [DyGu]. Let $(M, g)$ be a complete Riemannian manifold satisfying the assumptions of Theorem 1 . We put $\left(M_{1}, g_{1}\right)=$ $(M, g)$ and $\left(M_{0}, g_{0}\right)$ to be $\mathbb{R}^{n+1}$ with the Euclidean metric; let $N_{1}=N \subset M_{1}$ and $N_{0}=B\left(0, R_{0}\right) \subset M_{0}$, so that $\left(M_{1} \backslash N_{1}, g_{1}\right)$ is isometric to $\left(M_{0} \backslash N_{0}, g_{0}\right)$. We define $s(z)$ by duality like in (1.2) using the black-box trace: with the notation $\left[A_{j}\right]_{1}^{0}=A_{0}-A_{1}$, the black-box trace is defined by

$$
\begin{aligned}
\operatorname{Tr}_{\mathrm{bb}}\left(\varphi\left(\sqrt{\Delta_{g_{0}}}\right)-\varphi\left(\sqrt{\Delta_{g_{1}}}\right)\right):= & {\left[\operatorname{Tr}\left(\chi_{j} \varphi\left(\sqrt{\Delta_{g_{j}}}\right) \chi_{j}\right)\right]_{1}^{0}+\left[\operatorname{Tr}\left(\left(1-\chi_{j}\right) \varphi\left(\sqrt{\Delta_{g_{j}}}\right) \chi_{j}\right)\right]_{1}^{0} } \\
& +\left[\operatorname{Tr}\left(\chi_{j} \varphi\left(\sqrt{\Delta_{g_{j}}}\right)\left(1-\chi_{j}\right)\right)\right]_{1}^{0} \\
& +\operatorname{Tr}\left(\left[\left(1-\chi_{j}\right) \varphi\left(\sqrt{\Delta_{g_{j}}}\right)\left(1-\chi_{j}\right)\right]_{1}^{0}\right) .
\end{aligned}
$$

Here $\chi_{j} \in C_{0}^{\infty}\left(M_{j}\right)$ is a function which equals 1 near $N_{j}$, and $\left(1-\chi_{1}\right)=\left(1-\chi_{2}\right) \in$ $C^{\infty}\left(M_{j} \backslash N_{j}\right)$. All the traces exist by easy arguments, see [Sj97], and in the last trace, we use the identification $M_{1} \backslash N_{1} \sim M_{0} \backslash N_{0}$. The definition agrees with (1.1) when $M_{1}=M_{2}$. In this setting, $s(z)$ is always an analytic function on $[0, \infty)$, this can be seen for instance by using the Birman-Krein formula relating $s(z)$ with the argument of the determinant of the relative scattering operator (which is analytic and of modulus 1 on the real line).

Let us first recall Theorem 4 in [DyGu, Section 5.3]. We refer to [DiSj, Zw] or [DyGu, Section 3] for the definition of semiclassical pseudodifferential operators $\Psi^{*}(M)$ and semiclassical quantizations $\mathrm{Op}_{h}$.

Theorem 4. Let $(M, g)$ be a complete Riemannian manifold satisfying the assumptions of Theorem 1 and $\Lambda_{0}>\Lambda_{\max }$ with $\Lambda_{\max }$ defined in (1.4). Let $P(h)=h^{2} \Delta_{g}$ and let $A=$ $\mathrm{Op}_{h}(a) \in \Psi^{0}(M)$ be a compactly supported semi-classical pseudodifferential operator. Then there exist some differential operators $L_{j}$ of order $2 j$ on $T^{*} M$, depending on the quantization procedure $\mathrm{Op}_{h}$, with $L_{0}=1$, such that for all $L \in \mathbb{N}$, all $\lambda>0$, we have as $h \rightarrow 0$

$$
\operatorname{Tr}\left(A \mathbb{1}_{[0, \lambda]}(P(h))=(2 \pi h)^{-n-1} \sum_{j=0}^{L} h^{j} \int_{|\nu|_{g}^{2} \leq \lambda} L_{j} a d \mu_{\omega}+h^{-n} \mathcal{O}\left(\mu_{L}\left(\mathcal{T}\left(\Lambda_{0}^{-1}|\log h|\right)+h^{L}\right)\right.\right.
$$


where the remainder is uniform for $\lambda$ in a compact interval of $(0, \infty)$ and $\mu_{\omega}$ is the symplectic volume form on $T^{*} M$.

Using Theorem 4 and the commutator method of Robert [Ro], we will show Theorem 1.

Proof of Theorem 1. Define $\left(M_{1}, g_{1}\right)$ and $\left(M_{0}, g_{0}\right)$ as in the beginning of this section and put $\mathcal{E}=M_{1} \backslash N_{1}$, which is identified with $B^{c}:=M_{0} \backslash N_{0}$. Let $\chi_{j} \in C_{0}^{\infty}\left(M_{j}\right)$ be the cutoff functions used in the definition (2.1) of the black box trace. Let $\mathcal{A}$ be a smooth first order differential operator supported in $\mathcal{E}$, which is equal to $\mathcal{A}=\frac{1}{4}(m . \nabla+\nabla . m)$ on the support of $1-\chi_{1}$, where $m \in \mathbb{R}^{n+1}$ is the Euclidean coordinate in $\mathcal{E}$. A computation shows that $\left[\Delta_{g_{0}}, \mathcal{A}\right]=\Delta_{g_{0}}$ in $B^{c}$. Therefore, since $\Delta_{g_{1}}=\Delta_{g_{0}}$ on $\mathcal{E}$, we get, using the notation $\left[A_{j}\right]_{1}^{0}=A_{0}-A_{1}$,

$$
\begin{aligned}
\int_{0}^{\infty} \varphi\left(z^{2}\right) z^{2} \partial_{z} s(z) d z= & {\left[\operatorname{Tr}\left(\chi_{j} \Delta_{g_{j}} \varphi\left(\Delta_{g_{j}}\right)\right)\right]_{1}^{0}+\left[\operatorname{Tr}\left(\left(1-\chi_{j}\right) \Delta_{g_{j}} \varphi\left(\Delta_{g_{j}}\right) \chi_{j}\right)\right]_{1}^{0} } \\
& +\operatorname{Tr}\left(\left[\left(1-\chi_{j}\right)\left[\Delta_{g_{j}}, \mathcal{A}\right] \varphi\left(\Delta_{g_{j}}\right)\left(1-\chi_{j}\right)\right]_{1}^{0}\right)
\end{aligned}
$$

for all $\varphi \in C_{0}^{\infty}((0, \infty))$, and both traces make sense. Using the argument of Robert [Ro, Appendice A], it is direct to see by a limiting argument (using cutoff functions supported on large balls) and the cyclicity or the trace that

$$
\operatorname{Tr}\left(\left[\left(1-\chi_{j}\right)\left[\Delta_{g_{j}}, \mathcal{A}\right] \varphi\left(\Delta_{g_{j}}\right)\left(1-\chi_{j}\right)\right]_{1}^{0}\right)=\operatorname{Tr}\left(Q_{0} \varphi\left(\Delta_{g_{0}}\right)\right)-\operatorname{Tr}\left(Q_{1} \varphi\left(\Delta_{g_{1}}\right)\right)
$$

with $Q_{j}:=\left(\left(1-\chi_{j}\right)\left[\Delta_{g_{j}}, \chi_{j}\right]+\left[\Delta_{g_{j}}, \chi_{j}\right]\left(1-\chi_{j}\right)\right) \mathcal{A}$. Now we deduce that

$$
\int_{0}^{\infty} \varphi\left(z^{2}\right) z^{2} \partial_{z} s(z) d z=\operatorname{Tr}\left(S_{0} \varphi\left(\Delta_{g_{0}}\right)\right)-\operatorname{Tr}\left(S_{1} \varphi\left(\Delta_{g_{1}}\right)\right)
$$

with

$$
S_{j}:=Q_{j}+\chi_{j} \Delta_{g_{j}}+\chi_{j}\left(1-\chi_{j}\right) \Delta_{g_{j}}
$$

compactly supported second order differential operators. Let $\tilde{\chi}_{j} \in C_{0}^{\infty}\left(M_{j}\right)$ such that $\tilde{\chi}_{j}=1$ on $\operatorname{supp} \chi_{j}$, and in particular $\tilde{\chi}_{j} S_{j}=S_{j}$. We then obtain for $z>0$

$$
\partial_{z} s(z) d z=z^{-2}\left(\operatorname{Tr}\left(S_{0} d \Pi_{z^{2}}\left(\Delta_{g_{0}}\right) \widetilde{\chi}_{0}\right)-\operatorname{Tr}\left(S_{1} d \Pi_{z^{2}}\left(\Delta_{g_{1}}\right) \widetilde{\chi}_{1}\right)\right.
$$

where $d \Pi_{z}\left(\Delta_{g_{j}}\right)$ is the spectral measure of $\Delta_{g_{j}}$, i.e. such that $\varphi\left(\Delta_{g_{j}}\right)=\int_{0}^{\infty} \varphi(z) d \Pi_{z}\left(\Delta_{g_{j}}\right)$ for all $\varphi \in C_{0}^{\infty}(\mathbb{R})$. The traces here make sense since $d \Pi_{z^{2}}\left(\Delta_{g_{j}}\right)$ are operators with smooth kernels for $z>0$ (see e.g. [DyGu, Section 6.2]) and $S_{j}$ and $\tilde{\chi}_{j}$ are compactly supported. Let $\varepsilon>0$ be small and let $\psi \in C_{0}^{\infty}((1-\varepsilon, 1+\varepsilon))$ equal to 1 on $[1-\varepsilon / 2,1+\varepsilon / 2]$, and let $\varphi \in C_{0}^{\infty}((-1,1-\varepsilon / 2))$ be such that $\psi(\lambda)+\varphi(\lambda)=1$ for $0 \leq \lambda \leq 1$. Then for $h:=1 / z$ small

$$
\begin{aligned}
s(1 / h) & =\int_{0}^{1 / h} \psi\left(h^{2} u^{2}\right) \partial_{u} s(u) d u+\operatorname{Tr}_{\mathrm{bb}}\left(\varphi\left(h^{2} \Delta_{g_{0}}\right)-\varphi\left(h^{2} \Delta_{g_{1}}\right)\right) \\
& =\left[\operatorname{Tr}\left(T_{j} \mathbb{1}_{[0,1]}\left(h^{2} \Delta_{g_{j}}\right) \tilde{\chi}_{j}\right)\right]_{1}^{0}+\operatorname{Tr}_{\mathrm{bb}}\left(\varphi\left(h^{2} \Delta_{g_{0}}\right)-\varphi\left(h^{2} \Delta_{g_{1}}\right)\right)
\end{aligned}
$$


where $T_{j}:=h^{2} S_{j} \widetilde{\psi}\left(h^{2} \Delta_{g_{j}}\right)$ with $\widetilde{\psi}(\lambda)=\psi(\lambda) / \lambda$ in $C_{0}^{\infty}((1-\varepsilon, 1+\varepsilon))$. In all terms above, one can remove the functions $\widetilde{\chi}_{j}$ by cyclicity of the trace and using $\tilde{\chi}_{j} S_{j}=S_{j}$. Observe that $T_{j} \in \Psi^{-\infty}(M)$ by using the Helffer-Sjöstrand functional calculus [DiSj, Chapters 8-9]. Moreover, since $S_{j}$ is a compactly supported differential operator, $T_{j}$ can be decomposed into the sum of a compactly supported semi-classical pseudodifferential operator in $\Psi^{-\infty}(M)$ and of an operator $W_{j} \in h^{\infty} \Psi^{-\infty}(M)$ with Schwartz kernel $K_{W_{j}}\left(m, m^{\prime}\right)$ compactly supported in $m$ and decaying to infinite order as $m^{\prime} \rightarrow \infty$. Indeed, an operator in $\Psi^{-\infty}(M)$ has a smooth Schwartz kernel which is an $\mathcal{O}\left(\left(\frac{h}{d\left(m, m^{\prime}\right)}\right)^{\infty}\right)$ where $d\left(m, m^{\prime}\right)$ is the Riemannian distance (this comes from integration by parts in the oscillating integral defining the kernel). We then have

$$
\left|\operatorname{Tr}\left(W_{j} \mathbb{1}_{[0,1]}\left(h^{2} \Delta_{g_{j}}\right)\right)\right| \leq\left\|W_{j}\right\|_{\operatorname{Tr}}=\mathcal{O}\left(h^{\infty}\right)
$$

where $\|\cdot\|_{\operatorname{Tr}}$ is the trace norm. We can then apply Theorem 4 with the compactly supported operator $T_{j}-W_{j} \in \Psi^{-\infty}(M)$ instead of $T_{j}$ and we get an expansion in powers of $h$ for $\operatorname{Tr}\left(T_{1} \mathbb{1}_{[0,1]}\left(h^{2} \Delta_{g_{1}}\right)\right)$ up to $\mathcal{O}\left(h^{-n} \mu_{L}\left(\mathcal{T}\left(\Lambda_{0}^{-1}|\log h|\right)\right)\right)$, and a complete expansion for $\operatorname{Tr}\left(T_{0} \mathbb{1}_{[0,1]}\left(h^{2} \Delta_{g_{0}}\right)\right)$ since $g_{0}$ has no trapped set. The second term with $\varphi\left(h^{2} \Delta_{g_{j}}\right)$ has a full expansion by [Ro, Théorème 2.1], this is a consequence of the functional calculus for $h^{2} \Delta_{g_{j}}$, either using the Helffer-Sjöstrand method [DiSj, Chapters 8-9] or the Helffer-Robert [HeRo] approach. This achieves the proof.

In fact, this argument would apply similarly to any situation where the free Laplacian $\Delta_{g_{0}}$ on $\mathbb{R}^{n+1}$ is replaced by a self-adjoint operator $P_{0}$ satisfying the black-box setting [Sj97], with $P_{0}=\Delta_{g_{1}}$ on functions supported outside the black-box and for which there are expansions of $\operatorname{Tr}\left(A \Pi_{[0, z]}\left(P_{0}\right)\right)$ in powers of $z$ up to $\mathcal{O}\left(z^{n} \mu_{L}\left(\mathcal{T}\left(\Lambda_{0}^{-1} \log z\right)\right)\right)$ when $A$ is a compactly supported pseudo-differential operator.

2.2. Asymptotics using resonances near the real axis. For compact perturbations $P$ of the Euclidean Laplacian (this can be metric, obstacle or potential) in odd dimension $n+1$, the resolvent $R(z)=\left(P-z^{2}\right)^{-1}$ of $P$ extends meromorphically with poles of finite rank from $\{\operatorname{Im}(z)<0\}$ to $\mathbb{C}$, the poles are called resonances and form a discrete set $\mathcal{R} \subset\{\operatorname{Im}(z) \geq 0\}$. We associate to each resonance $z_{j}$ its multiplicity $m_{j} \in \mathbb{N}$ as in [SjZw91]. The following sharp upper bounds on the counting function of resonances for metric perturbations have been proved by Sjöstrand-Zworski [SjZw91] and Vodev [Vo]

$$
\sharp\{\rho \in \mathcal{R} ;|\rho| \leq R\}=\mathcal{O}\left(R^{n+1}\right) .
$$

Proof of Theorem 2. Let $\chi \in C_{0}^{\infty}(M)$ be a function which is equal to 1 near $N$, and define the tempered distribution on $\mathbb{R}$

$$
u(t):=2 \operatorname{Tr}\left(\cos \left(t \sqrt{\Delta_{g}}\right)-(1-\chi) \cos \left(t \sqrt{\Delta_{\mathbb{R}^{n+1}}}\right)(1-\chi)\right) .
$$




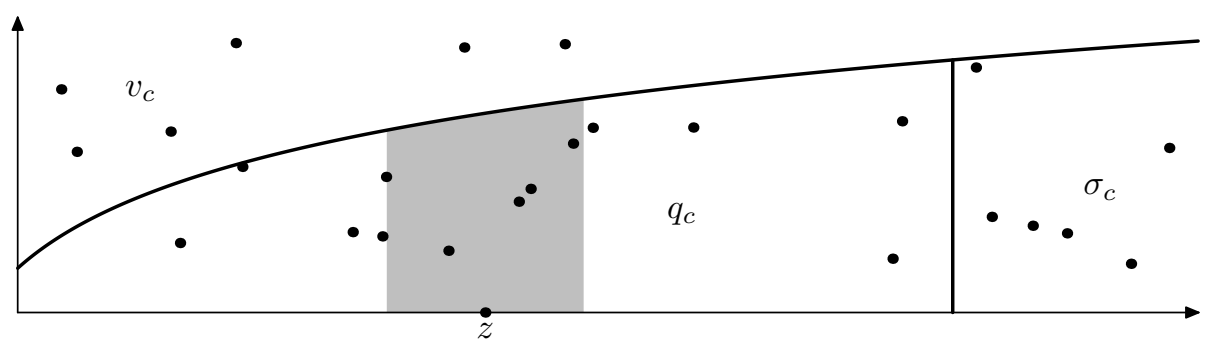

FiguRE 1. The sets of resonances used in the proof of Theorem 2. We mark the regions corresponding to the sums $v_{c}, q_{c}$, and $\sigma_{c} . \mathcal{R}_{c}$ is the region below the curve, while the shaded region corresponds to $\mathcal{N}_{c, \log }(z)$.

By a straightforward computation, for all $\chi \in C_{0}^{\infty}\left(\mathbb{R}^{n+1}\right), \operatorname{Tr}\left(\chi \cos \left(t \sqrt{\Delta_{\mathbb{R}^{n+1}}}\right)\right)$ is a distribution supported at $t=0$ and its Fourier transform is a polynomial of order $n$. Thus, using the definition (2.1) of the black-box trace, we easily see that there exists a polynomial $P_{n}$ of degree $n$ such that for all $\varphi \in C_{0}^{\infty}((0, \infty))$

$$
\frac{1}{2 \pi}\langle\hat{u}, \varphi\rangle=\operatorname{Tr}_{\mathrm{bb}}\left(\varphi\left(\sqrt{\Delta_{g}}\right)-\varphi(\sqrt{\Delta})\right)+\left\langle P_{n}, \varphi\right\rangle=-\int_{0}^{\infty}\left(\partial_{z} s(z)-P_{n}(z)\right) \varphi(z) d z .
$$

It then suffices to study the asymptotics of $\int_{0}^{z} \hat{u}(w) d w$ as $z \rightarrow \infty$. In [SjZw94, Theorem 2], Sjöstrand-Zworski proved the following Poisson formula extending [Me83] (see also [Zw97, Theorem 2] for a direct proof without using Lax-Phillips theory)

$$
u(t)=\sum_{\rho \in \mathcal{R}} e^{i \rho|t|}, \quad t \neq 0 .
$$

Moreover, $u(t)$ is classically conormal at $t=0$, this follows from writing the wave propagator as a (classical) Fourier Integral Operator (see e.g. [HöIII, Sections 17.4 and 17.5]): if $\theta \in C_{0}^{\infty}\left(-t_{0}+\delta, t_{0}-\delta\right)$ for $\delta>0$ small enough, $\theta(t) \in[0,1]$ and $\theta(t)=1$ in $\left[-t_{0}+2 \delta, t_{0}-2 \delta\right]$, then

$$
\mathcal{F}(u(t) \theta(t))(z) \sim \sum_{k=0}^{\infty} a_{k}^{ \pm} z^{n-k} \text { as } z \rightarrow \pm \infty
$$

for some coefficients $a_{k}^{ \pm}$(which are integrals of local invariants in the metric $g$ ). Here $\mathcal{F}$ denotes the Fourier transform and $t_{0}$ is the injectivity radius of $M$. Notice that, by (2.2), if $L$ is large enough, $t^{L} \sum_{\rho \in \mathcal{R}} e^{i \rho|t|}$ is a well defined distribution on $\mathbb{R}$ and it equals $t^{L} u(t)$ (in fact $L$ can be taken to be $n$, see [Zw97, Theorem 7]).

Let $c>0$ and define the region (see Figure 1)

$$
\mathcal{R}_{c}:=\{\rho \in \mathcal{R} ; \operatorname{Im}(\rho) \leq 1+c \log |\operatorname{Re}(\rho)|\} .
$$

We decompose $u(t)(1-\theta(t))$ as

$$
u(t)(1-\theta(t))=u_{c}(t)+v_{c}(t), \quad \text { with } u_{c}(t)=\sum_{\rho \in \mathcal{R}_{c}} e^{i \rho|t|}(1-\theta(t)) .
$$


Both terms are tempered distributions on $\mathbb{R}$. From $(2.2)$, one has $v_{c}(t) \in L^{p}(\mathbb{R})$ for all $p>1$ if $c t_{0}>n+1$ and $\delta$ small enough: indeed

$$
\left|v_{c}(t)\right| \leq \sum_{\rho \in \mathcal{R} \backslash \mathcal{R}_{c}}|1-\theta(t)| e^{-\operatorname{Im}(\rho)|t|} \leq C(1-\theta(t)) \int_{1}^{\infty} R^{-c|t|+n} d R \leq \frac{C(1-\theta(t))}{c|t|-n-1} .
$$

This inequality also implies $\left|v_{c}(t) / t\right| \leq C\left(1+t^{2}\right)^{-1}$ and therefore we get $\mathcal{F}\left(v_{c}(t) / t\right) \in$ $L^{\infty}(\mathbb{R}) \cap C^{0}(\mathbb{R})$. We thus obtain

$$
\int_{0}^{z} \hat{v}_{c}(w) d w=i \mathcal{F}\left(\frac{v_{c}(t)}{t}\right)(z)-i \int \frac{v_{c}(t)}{t} d t \in L^{\infty}(\mathbb{R}) .
$$

In particular, the left-hand side of $(2.4)$ is $\mathcal{O}\left(z^{\alpha}\right)$ as $z \rightarrow \infty$.

It remains to consider the Fourier transform of $u_{c}$. Let $\psi \in C_{0}^{\infty}((-2,2))$ be equal to 1 on $(-1,1)$, then we are going to show that

$$
\begin{gathered}
\hat{u}_{c}(z)=2 \pi \sigma_{c}(z)-\sigma_{c} \star \hat{\theta}(z)+2 \pi q_{c}(z)-q_{c} \star \hat{\theta}(z), \\
\text { with } q_{c}(z):=\sum_{\rho \in \mathcal{R}_{c}} \psi\left(\frac{|\rho|}{z}\right) \frac{2 \operatorname{Im}(\rho)}{|\rho-z|^{2}}
\end{gathered}
$$

and $\sigma_{c}(z)$ a symbol, that is there exists $L^{\prime}$ such that for all $k \in \mathbb{N}$, there is $C_{k}>0$ such that for all $z \in \mathbb{R}$

$$
\left|\partial_{z}^{k} \sigma_{c}(z)\right| \leq C_{k}(1+|z|)^{L^{\prime}-k}
$$

The proof follows closely the argument of Melrose [Me88]. We have as tempered distribution

$$
\mathcal{F}\left(e^{i \rho|t|}+e^{-i \bar{\rho}|t|}\right)(z)=\frac{2 \operatorname{Im}(\rho)}{|z-\rho|^{2}}+\frac{2 \operatorname{Im}(-\bar{\rho})}{|z+\bar{\rho}|^{2}}
$$

and since the resonances are symmetric with respect to the imaginary axis, we have

$$
\mathcal{F}\left(t^{L} \sum_{\rho \in \mathcal{R}_{c}} e^{i \rho|t|}\right)(z)=\sum_{\rho \in \mathcal{R}_{c}}\left(i \partial_{z}\right)^{L}\left(\frac{2 \operatorname{Im}(\rho)}{|z-\rho|^{2}}\right)
$$

and these make sense as tempered distribution on $\mathbb{R}$ by (2.2). Then

$$
\left(i \partial_{z}\right)^{L}\left(\hat{u}_{c}(z)-q_{c} \star\left(2 \pi \delta_{0}-\hat{\theta}\right)(z)\right)=\sum_{\rho \in \mathcal{R}_{c}}\left(i \partial_{z}\right)^{L}\left(\left(1-\psi\left(\frac{|\rho|}{\cdot}\right)\right) \frac{2 \operatorname{Im}(\rho)}{|\cdot-\rho|^{2}}\right) \star\left(2 \pi \delta_{0}-\hat{\theta}\right)(z)
$$

and one can easily see that

$$
\sigma_{c, L}(z):=\sum_{\rho \in \mathcal{R}_{c}}\left(i \partial_{z}\right)^{L}\left(\left(1-\psi\left(\frac{|\rho|}{z}\right)\right) \frac{2 \operatorname{Im}(\rho)}{|z-\rho|^{2}}\right)
$$

is a symbol (see [Me88] for details) if $L$ is large enough. Integrate $\sigma_{c, L}(z) L$ times, this defines a classical symbol with an order increasing by $L$, that we denote $\sigma_{c}(z)$ and such that $\left(i \partial_{z}\right)^{L}\left(\sigma_{c}(z)\right)=\sigma_{c, L}(z)$, and we therefore deduce that

$$
\hat{u}_{c}(z)-q_{c} \star\left(2 \pi \delta_{0}-\hat{\theta}\right)(z)-\sigma_{c} \star\left(2 \pi \delta_{0}-\hat{\theta}\right)(z)=P_{L}(z)
$$


where $P_{L}(z)$ is a polynomial of order $L$. Taking inverse Fourier transform of the left hand side give a distribution vanishing near $t=0$ and therefore $P_{L}(z)=0$ necessarily. This shows (2.5). Since for $z$ large $2 \pi \sigma_{c}(z)-\sigma_{c} \star \hat{\theta}(z)=\mathcal{O}\left(z^{-\infty}\right)$, we also get

$$
\hat{u}_{c}(z)=q_{c} \star\left(2 \pi \delta_{0}-\hat{\theta}\right)(z)+\mathcal{O}\left(z^{-\infty}\right) .
$$

An easy computation yields

$$
\forall a \leq b, \quad \int_{a}^{b} \frac{\operatorname{Im}(\rho)}{|z-\rho|^{2}} d z \leq \pi
$$

and if $\operatorname{Re}(\rho)+1<a<b$ or $a<b<\operatorname{Re}(\rho)-1$

$$
\int_{a}^{b} \frac{\operatorname{Im}(\rho)}{|z-\rho|^{2}} d z \leq \frac{(b-a) \operatorname{Im}(\rho)}{(b-\operatorname{Re}(\rho))(a-\operatorname{Re}(\rho))} .
$$

Define $\mathcal{N}_{c, \log }(z):=\sharp\left\{\rho \in \mathcal{R}_{c} ;|z-\operatorname{Re}(\rho)| \leq \log (z)\right\}$ and set $Q_{c}(z)=\int_{0}^{z} q_{c}(w) d w$. Then we obtain from (2.7) and (2.8) that for $w \in[0,1]$ and $z \gg 1$

$$
\left|Q_{c}(z)-Q_{c}(z-w)\right| \leq C\left(\mathcal{N}_{c, \log }(z)+\sum_{\substack{\rho \in \mathcal{R}_{c},|\rho| \leq 2 z \\ \operatorname{Re}(\rho)-z \mid>\log (z)}} \frac{\operatorname{Im}(\rho)}{(z-\operatorname{Re}(\rho))^{2}}\right) .
$$

Using the assumption $\mathcal{N}_{c, \log }(z)=\mathcal{O}\left(z^{\alpha}\right)$, we easily deduce for $z \gg 1$ and $w \in[0,1]$

$$
\left|Q_{c}(z)-Q_{c}(z-w)\right|=\mathcal{O}\left(|z|^{\alpha}\right) .
$$

Thus iterating and using the same argument for $z<0$, we get for all $z, t \in \mathbb{R}$

$$
\left|Q_{c}(z)-Q_{c}(z-w)\right| \leq C(1+|w|)(1+|z|+|w|)^{\alpha} .
$$

We multiply by $\hat{\theta}(t)$ and integrate to deduce

$$
\left|2 \pi Q_{c}(z)-Q_{c} \star \hat{\theta}(z)\right| \leq C(1+|z|)^{\alpha} .
$$

It follows by (2.6) that

$$
\left|\int_{0}^{z} \hat{u}_{c}(w) d w\right| \leq C(1+|z|)^{\alpha} .
$$

Combining this with (2.4) and (2.3), this yields that as $z \rightarrow \infty$

$$
\int_{0}^{z} \hat{u}(w) d w=\sum_{j=0}^{n+1} c_{j} z^{n+1-j}+\mathcal{O}\left(z^{\alpha}\right)
$$

for some $c_{j} \in \mathbb{R}$. This achieves the proof.

Note that the proof applies for obstacles since in that case the small time asymptotics of the wave trace is proved by Ivrii [Iv]. 


\section{SCATtering PhASE ASYMPtotics FOR HYPERBolic QUOTIENTS}

3.1. Convex co-compact groups. A convex co-compact group $\Gamma$ of isometries of hyperbolic space $\mathbb{H}^{n+1}$ is a discrete group of hyperbolic transformations (their fixed points on $\overline{\mathbb{B}}=\left\{m \in \mathbb{R}^{n+1} ;|m| \leq 1\right\}$ are 2 disjoint points on $\mathbb{S}^{n}=\partial \bar{M}$ ) with a compact convex core. The limit set $\Lambda_{\Gamma}$ of the group and the discontinuity set $\Omega_{\Gamma}$ are defined by

$$
\Lambda_{\Gamma}:=\overline{\left\{\gamma(m) \in \mathbb{H}^{n+1} ; \gamma \in \Gamma\right\}} \cap \mathbb{S}^{n}, \quad \Omega_{\Gamma}:=\mathbb{S}^{n} \backslash \Lambda_{\Gamma}
$$

where the closure is taken in the closed unit ball $\overline{\mathbb{B}}$ and $m \in \mathbb{H}^{n+1}$ is any point. The group $\Gamma$ acts on the convex hull of $\Lambda_{\Gamma}$ (with respect to hyperbolic geodesics) and the convex core is the quotient space. The group $\Gamma$ acts properly discontinuously on $\Omega_{\Gamma}$ as a group of conformal transformation and for convex co-compact groups, the quotient $\Gamma \backslash \Omega_{\Gamma}$ is compact. The manifold $M=\Gamma \backslash \mathbb{H}^{n+1}$ admits a natural smooth compactification given by $\bar{M}=\Gamma \backslash\left(\mathbb{H}^{n+1} \cup \Omega_{\Gamma}\right)$, where the smooth structure is induced by that of the closed unit ball $\overline{\mathbb{B}}$ compactifying $\mathbb{H}^{n+1}$. The hyperbolic metric on $M$ is asymptotically hyperbolic in the sense of Mazzeo-Melrose [MaMe]. Let $x$ be a nonnegative function which is a boundary defining function for $\partial \bar{M}=\Gamma \backslash \Omega_{\Gamma}$. We say that $x$ is a geodesic boundary defining function if $|d \log x|=1$ near $\partial \bar{M}$, where the norm is taken with respect to the hyperbolic metric on $M$. Such functions exist, this is proved for instance by Graham [Gr, Lemma 2.1].

An important quantity is the Hausdorff dimension of $\Lambda_{\Gamma}$

$$
\delta:=\operatorname{dim}_{\mathrm{H}} \Lambda_{\Gamma}<n
$$

which in turn is, by Patterson [Pa] and Sullivan [Su], the exponent of convergence of Poincaré series

$$
m \in \mathbb{H}^{n+1}, \quad \sum_{\gamma \in \Gamma} e^{-s d(m, \gamma m)}<\infty \Longleftrightarrow s>\delta .
$$

where here and below, $d(\cdot, \cdot)$ is the distance for the hyperbolic metric on $\mathbb{H}^{n+1}$. Note that the series is locally uniformly bounded in $m \in \mathbb{H}^{n+1}$. The Hausdorff dimension of the trapped set $K$ of the geodesic flow on $\Gamma \backslash \mathbb{H}^{n+1}$ is given by $2 \delta+1$, see Sullivan [Su].

3.2. Krein's type spectral function. In [Gu, Theorem 1.3], the second author defined on even dimensional convex co-compact hyperbolic manifolds $M=\Gamma \backslash \mathbb{H}^{n+1}$ a generalized Krein function $\xi(z)$, which is a regularized trace of the spectral projector $\Pi_{\left[n^{2} / 4, n^{2} / 4+z^{2}\right]}(\Delta)$, and replaces the spectral shift function in this setting. As we will see, it is related to the scattering phase. It is defined as follows

$$
\xi(z)=\int_{0}^{z^{2}} \operatorname{Tr}_{R}\left(d \Pi_{u}(\Delta)\right), \quad z>0
$$

where $d \Pi_{u}(\Delta)$ is the spectral measure of the Laplacian, i.e. $\int_{0}^{z} d \Pi_{u}(\Delta)=\mathbb{1}_{\left[\frac{n^{2}}{4}, \frac{n^{2}}{4}+u\right]}(\Delta)$, and the regularized trace is defined using Hadamard finite part: let $x$ be a geodesic 
boundary function in $\bar{M}$, then if $T$ is an operator with smooth integral kernel $T\left(m, m^{\prime}\right)$ such that the restriction to the diagonal $m \mapsto T(m, m)$ has an asymptotic expansion at $\partial \bar{M}$ in powers of $x(m)$, we set

$$
\operatorname{Tr}_{R}(T)=\mathrm{FP}_{\varepsilon \rightarrow 0} \int_{x>\varepsilon} T(m, m) \operatorname{dvol}_{g}(m)
$$

and FP stands for finite part (ie. the coefficient of $\varepsilon^{0}$ in the expansion as $\varepsilon \rightarrow 0$ ). The renormalized trace should a priori depend on the choice of $x$ but in fact $\operatorname{Tr}_{R}\left(d \Pi_{u}(\Delta)\right)$ does not depend on $x$, see [Gu]. It is natural to use this definition in this setting as there is no model operator to compare with, unlike for perturbations of Euclidean space. The function $\xi^{\prime}(z)$ has meromorphic extension to $z \in \mathbb{C}$ and $\xi$ is a phase of a regularized determinant of the scattering operator $S(n / 2+i z)$, see [Gu, Theorem 1.2]. The following identity is proved in [Gu, Theorem 1.3] when $n+1$ is even $(\Gamma(\lambda)$ is the Euler function)

$$
\frac{Z_{\Gamma}\left(\frac{n}{2}-i z\right)}{Z_{\Gamma}\left(\frac{n}{2}+i z\right)}=\exp \left(-2 i \pi \xi(z)+\chi(M) \frac{2 i \pi(-1)^{\frac{n+1}{2}}}{\Gamma(n+1)} \int_{0}^{z} \frac{\Gamma\left(\frac{n}{2}+i t\right) \Gamma\left(\frac{n}{2}-i t\right)}{\Gamma(i t) \Gamma(-i t)} d t+c i \pi\right)
$$

where $c \in \mathbb{N}$ is a constant given by the multiplicity of $n / 2$ as a resonance, $\chi(M)$ is the Euler characteristic, $Z_{\Gamma}(\lambda)$ is the Selberg zeta function, defined for $\Re(\lambda)>\delta$ (here $\delta$ is defined in (3.2))

$$
Z_{\Gamma}(\lambda)=\exp \left(-\sum_{\gamma} \sum_{m=1}^{\infty} \frac{1}{m} \frac{e^{-\lambda m l(\gamma)}}{G_{\gamma}(m)}\right)
$$

where $\gamma$ runs over the set $\mathcal{L}$ of primitive closed geodesics of $X, \ell(\gamma)$ is the length of $\gamma$ and $G_{\gamma}(m):=e^{-\frac{n}{2} m l(\gamma)}\left|\operatorname{det}\left(1-P_{\gamma}^{m}\right)\right|^{\frac{1}{2}}$ if $P_{\gamma}$ is the Poincaré linear map associated to the primitive periodic orbit $\gamma$ of the geodesic flow on the unit tangent bundle. The Selberg zeta function extends to $\mathbb{C}$ and has only finitely many zeros in $\left\{\operatorname{Re}(\lambda) \geq \frac{n}{2}\right\}$, all on the real line by Patterson-Perry [PaPe]. The zeros of $Z_{\Gamma}(\lambda)$ in $\{\operatorname{Re}(\lambda)<n / 2\}$ are the poles of the meromorphic continuation of the resolvent $R(\lambda)=(\Delta-\lambda(n-\lambda))^{-1}$ of the Laplacian from $\{\operatorname{Re}(\lambda)>n / 2\}$ to $\mathbb{C}$ (as a map $C_{0}^{\infty}(M) \rightarrow C^{\infty}(M)$ ). Keeping the convention in Section 2.2 for resonances, we say that $\rho \in\{\operatorname{Im}(\rho) \geq 0\}$ is a resonance if this is a pole of the meromorphic continuation of $z \rightarrow\left(\Delta-n^{2} / 4-z^{2}\right)^{-1}$ from the lower half plane to $\mathbb{C}$. The function with Gamma factors can be written as

$$
\frac{\Gamma\left(\frac{n}{2}+i z\right) \Gamma\left(\frac{n}{2}-i z\right)}{\Gamma(i z) \Gamma(-i z)}=\left(\frac{1}{4}+z^{2}\right) \ldots\left(\left(\frac{n}{2}-1\right)^{2}+z^{2}\right) z\left(1+\mathcal{O}\left(z^{-\infty}\right)\right)
$$

as $z \rightarrow \infty$. Notice that this is an odd polynomial modulo $\mathcal{O}\left(z^{-\infty}\right)$. Since $Z_{\Gamma}(\bar{\lambda})=$ $\overline{Z_{\Gamma}(\lambda)}$, we deduce that for $z>0$,

$$
\xi(z)=\chi(M) F_{n+1}(z)+\frac{1}{\pi} \operatorname{Arg}\left(Z_{\Gamma}(n / 2+i z)\right)+\frac{1}{2} c
$$


where $2 i \pi F_{n+1}(z)$ is the explicit function in factor of $\chi(M)$ in (3.5) (this is a polynomial modulo $\left.\mathcal{O}\left(z^{-\infty}\right)\right)$, and $\operatorname{Arg}\left(Z_{\Gamma}(n / 2+i z)\right)$ is defined by

$$
\operatorname{Arg}\left(Z_{\Gamma}(n / 2+i z)\right)=\int_{0}^{z} \operatorname{Re}\left(\frac{Z_{\Gamma}^{\prime}\left(\frac{n}{2}+i t\right)}{Z_{\Gamma}\left(\frac{n}{2}+i t\right)}\right) d t
$$

The case $\delta<n / 2$. The series defining $Z_{\Gamma}\left(\frac{n}{2}+i z\right)$ converges uniformly for $z \in \mathbb{R}$ and therefore one obtains directly an explicit formula (in this case $c=0$ as there is no resonance at $n / 2$ )

$$
\xi(z)=\chi(M) \frac{(-1)^{\frac{n+1}{2}}}{\Gamma(n+1)} \int_{0}^{z} \frac{\Gamma\left(\frac{n}{2}+i t\right) \Gamma\left(\frac{n}{2}-i t\right)}{\Gamma(i t) \Gamma(-i t)} d t+\frac{1}{\pi} \sum_{\gamma} \sum_{m=1}^{\infty} \frac{e^{-\frac{n}{2} m \ell(\gamma)} \sin (z \ell(\gamma))}{m G_{\gamma}(m)} .
$$

The last term in the right hand side is $\mathcal{O}(1)$ as $z \rightarrow \infty$, and by Gauss-Bonnet, we notice that $\chi(M)$ can be written as a constant times a regularized volume $\operatorname{Vol}_{R}(M)$ of $M$, defined by $\operatorname{Vol}_{R}(M)=\mathrm{FP}_{\varepsilon \rightarrow 0} \int_{x>\varepsilon} 1 \operatorname{dvol}_{g}(m)$ (cf [PaPe, Appendix A]), corresponding to the usual Weyl type asymptotics for spectral shift functions. The classical dynamic only appears at order $z^{0}$ (with oscillations) in the asymptotic.

The case $\delta \geq n / 2$. We can not use the convergence of $Z_{\Gamma}\left(\frac{n}{2}+i z\right)$ anymore. We know by Guillopé-Lin-Zworski [GuLiZw] that for Schottky groups $\Gamma$ (in particular all convex co-compact groups in dimension $n+1=2$ ), one has for any $\sigma_{0}$, that there exists $C_{\sigma_{0}}>0$ such that for all $z>1$ and $\sigma>\sigma_{0}$

$$
\log \left|Z_{\Gamma}(\sigma+i z)\right| \leq C_{\sigma_{0}} z^{\delta}
$$

Lemma 3.1. There exists $N>0$ such that for all $z \in \mathbb{R}, \operatorname{Re}\left(Z_{\Gamma}(N+i z)\right)>1 / 2$.

Proof. From the definition of $Z_{\Gamma}(\lambda)$ for $\operatorname{Re}(\lambda)>\delta$, we have

$$
\operatorname{Re}\left(Z_{\Gamma}(\lambda)\right)=\exp \left(-\sum_{m=1}^{\infty} \frac{1}{m} \operatorname{Re}\left(T_{\lambda}(m)\right)\right) \cos \left(\sum_{m=1}^{\infty} \frac{1}{m} \operatorname{Im}\left(T_{\lambda}(m)\right)\right)
$$

where $T_{\lambda}(m)=\sum_{\gamma} e^{-\lambda m \ell(\gamma)} / G_{\gamma}(m)$. Let $\lambda=N+i z$ and we shall take $N$ large. We claim that there is $N$ large enough and $C>0$ such that for all $m \in \mathbb{N}, z \in \mathbb{R}$

$$
\left|T_{N+i z}(m)\right| \leq C e^{-N m \ell\left(\gamma_{0}\right)} .
$$

where $\gamma_{0}$ is the geodesic with the smallest length. This follows directly from a lower bound $\left|G_{\gamma}(m)\right| \geq c_{0}$ for some $c_{0}>0$ independent of $m$ and $\gamma$, and the estimate

$$
\sharp\{\gamma \in \mathcal{L} ; \ell(\gamma) \leq R\}=\mathcal{O}\left(e^{\delta R}\right), \quad R \rightarrow \infty .
$$

To get the lower bound of $\left|G_{\gamma}(m)\right|$, it suffices to write this term explicitly: associated to the primitive geodesic $\gamma$ there is a conjugacy class of hyperbolic isometries in $\Gamma$, and $\gamma$ is conjugated by a hyperbolic isometry to the transformation $(x, y) \mapsto e^{\ell(\gamma)}\left(O_{\gamma}(x), y\right)$, 
in the half space $(x, y) \in \mathbb{H}^{n+1}=\mathbb{R}^{n} \times \mathbb{R}_{+}$where $O_{\gamma} \in S O_{n}(\mathbb{R})$. Denoting by $\alpha_{1}(\gamma), \ldots, \alpha_{n}(\gamma)$ the eigenvalues of $O_{\gamma}$, then

$$
G_{\gamma}(m)=\operatorname{det}\left(I-e^{-m \ell(\gamma)} O_{\gamma}^{m}\right)=\prod_{i=1}^{n}\left(1-e^{-m \ell(\gamma)} \alpha_{i}(\gamma)^{m}\right) .
$$

Therefore $\left|G_{\gamma}(m)\right| \geq\left(1-e^{-m \ell\left(\gamma_{0}\right)}\right)^{n} \geq\left(1-e^{-\ell\left(\gamma_{0}\right)}\right)^{n}$ where $\ell\left(\gamma_{0}\right)>0$ is the length of the shortest geodesic on $M$.

We conclude that for any $\varepsilon>0$, there is $C>0$ such that for all $z \in \mathbb{R}$

$$
\sum_{m=1}^{\infty} \frac{1}{m}\left|T_{N+i z}(m)\right| \leq C e^{-N\left(\ell\left(\gamma_{0}\right)-\varepsilon\right)}
$$

and taking $N$ large enough and $\varepsilon$ small enough, we deduce the Lemma.

One can obtain an approximation of the argument of $Z_{\Gamma}\left(\frac{n}{2}+i z\right)$ in terms of its zeros, this follows from a general result on holomophic functions:

Lemma 3.2. Assume that $\Gamma$ is a Schottky group in dimension $n+1$ even with Hausdorff dimension of limit set $\delta$. Then for all $\sigma>0$ fixed, there is a constant $C>0$ depending on $\sigma$ such that for all $z>0$ large and all $\lambda$ in the disc

$$
D(n / 2+i z, \sigma):=\{\lambda \in \mathbb{C} ;|\lambda-n / 2-i z| \leq \sigma\}
$$

we have the identity

$$
\left|\frac{Z_{\Gamma}^{\prime}(\lambda)}{Z_{\Gamma}(\lambda)}-\sum_{s \in \mathcal{R}_{\sigma+1}} \frac{1}{\lambda-s}\right| \leq C z^{\delta}
$$

where $\mathcal{R}_{\sigma+1}$ is the set of zeros of $Z_{\Gamma}(\lambda)$ in $D(n / 2+i z, \sigma+1)$.

Proof. We apply Lemma $\alpha$ of [Ti, Section 3.9] with the holomorphic function $Z_{\Gamma}(\lambda)$ in a disc $D(N+i z, 2 N)$ where $N>0$ is a large fixed parameter (chosen independent of $z$ ). Using the bound $\left|Z_{\Gamma}(\lambda)\right| \leq e^{C z^{\delta}}$ of [GuLiZw] for $\lambda \in D(N+i z, 8 N)$ where $C$ depends on $N$, and the lower bound $\left|Z_{\Gamma}(N+i z)\right|>1 / 2$ for all $z>0$ if $N$ is large enough by Lemma 3.1, we obtain that there exists $C^{\prime}$ depending on $C, N$ such that for all $\lambda \in D(N+i z, 2 N)$

$$
\left|\frac{Z_{\Gamma}^{\prime}(\lambda)}{Z_{\Gamma}(\lambda)}-\sum_{s \in \mathcal{R}_{N}} \frac{1}{\lambda-s}\right| \leq C^{\prime} z^{\delta}
$$

where $\mathcal{R}_{N}$ is the set of zeros of $Z_{\Gamma}(\lambda)$ in the disc $D(N+i z, 4 N)$. To reduce to a sum over $\mathcal{R}_{\sigma+1}$, it thus suffices to use that the number of zeros of $Z_{\Gamma}(\lambda)$ in $D(N+i z, 4 N)$ is bounded by $C^{\prime \prime} z^{\alpha}$ for some $C^{\prime \prime}$ depends only on $N$ (by [GuLiZw] again), and $|\lambda-s| \geq 1$ if $|\lambda-n / 2-i z| \leq \sigma$ and $s \in \mathcal{R}_{N} \backslash \mathcal{R}_{\sigma+1}$. This achieves the proof.

We also have an estimate on the argument of $Z_{\Gamma}(n / 2+i z)$ by using another Lemma in Titschmarch's book [Ti, Section 9.4]. 
Lemma 3.3. Fix $N>\sigma_{0}>0$. Let $f(z)$ be a holomorphic function in $\{\operatorname{Re}(z)>0\}$, with $f(z) \in \mathbb{R}$ for $z \in \mathbb{R}$ and there exists $K>0$ such that $|\operatorname{Re}(f(N+i t))| \geq K$ for all $t \in \mathbb{R}$. Suppose that $\left|f\left(\sigma^{\prime}+i t^{\prime}\right)\right| \leq M_{\sigma, t}$ for all $1 \leq t^{\prime} \leq t$ and $\sigma^{\prime} \geq \sigma$ with $\sigma \geq \sigma_{0}$. Then if $T$ is not the imaginary part of a zero of $f$, we have

$$
|\operatorname{Arg}(f(\sigma+i T))| \leq C\left(\log \left(M_{\sigma_{0}, T+2}\right)-\log (K)\right)+3 \pi / 2
$$

for some $C>0$ depending only on $\sigma_{0}$ and $N$.

We apply this to the function $Z_{\Gamma}(z)$ by using Lemma 3.1 and (3.8), we obtain directly

Corollary 3.4. If $\Gamma$ is a convex co-compact Schottky group of orientation preserving isometries of $\mathbb{H}^{n+1}$ with $n+1$ is even, and $Z_{\Gamma}(\lambda)$ the Selberg zeta function for $\Gamma$, then for $z>1$ large

$$
\operatorname{Arg}\left(Z_{\Gamma}(n / 2+i z)\right)=\mathcal{O}\left(z^{\delta}\right)
$$

where $\delta>0$ is the Hausdorff dimension of the limit set of $\Gamma$.

In fact an integrated estimate is also true, by using a lemma of Hadamard:

Lemma 3.5. Let $\Gamma$ be a convex co-compact Schottky group of orientation preserving isometries of $\mathbb{H}^{n+1}$ with $n+1$ is even, and $Z_{\Gamma}(\lambda)$ the Selberg zeta function for $\Gamma$ and $\delta>0$ the Hausdorff dimension of the limit set. Then for $T>1$ large

$$
\int_{1}^{T} \operatorname{Arg}\left(Z_{\Gamma}(n / 2+i z)\right) d t=\mathcal{O}\left(T^{\delta}\right) .
$$

Proof. Let $T>1$. We apply the Hadamard Lemma to the holomorphic function $Z_{\Gamma}(n / 2-i z)$ in the rectangle $z \in[1, T]+i[0, N]$, with no zeros in that rectangle: we get

$$
\begin{gathered}
\int_{0}^{N} \log \left|Z_{\Gamma}(n / 2+u-i)\right| d u-\int_{0}^{N} \log \left|Z_{\Gamma}(n / 2+u-i T)\right| d u= \\
\int_{1}^{T} \operatorname{Arg}\left(Z_{\Gamma}(n / 2-i z)\right) d z-\int_{1}^{T} \operatorname{Arg}\left(Z_{\Gamma}(N-i z)\right) d z .
\end{gathered}
$$

Now, $\left|\operatorname{Arg}\left(Z_{\Gamma}(N-i z)\right)\right|$ is bounded independently of $z$ when $N$ is large enough, thus the estimate (3.8) gives the result when $\delta \geq 1$. When $\delta<1$, we take $N \sim c \log (T)$ with $c>4 / \ell\left(\gamma_{0}\right)$ and use that $\log \left|Z_{\Gamma}(n / 2+u-i z)\right| \leq C z^{\delta}$ in $u \in\left[0, u_{0}\right]$. By what we explained above, if $u_{0}$ is large enough (and we take it independent of $T$ ), we also have for all $z \in \mathbb{R}$ and $u>u_{0}$

$$
\left|\log \left(Z_{\Gamma}(n / 2+u-i z)\right)\right| \leq C e^{-u \ell\left(\gamma_{0}\right) / 2}
$$

thus

$$
\begin{aligned}
\int_{0}^{N} \log \left|Z_{\Gamma}(n / 2+u-i)\right| d u+|\log | Z_{\Gamma}(n / 2+u-i T) \| d u & \leq C T^{\delta}+\int_{u_{0}}^{N} e^{-u \ell\left(\gamma_{0}\right) / 2} d u \\
& \leq C T^{\delta}
\end{aligned}
$$


and

$$
\int_{1}^{T}\left|\operatorname{Arg}\left(Z_{\Gamma}(N-i z)\right)\right| d z \leq \int_{1}^{T} e^{-N \ell\left(\gamma_{0}\right) / 2} d t \leq C T^{-1}
$$

Gathering all these results, we obtain (we assume $\delta \geq n / 2$ as otherwise one has a complete formula by (3.7))

Theorem 5. Let $\Gamma$ be a convex co-compact Schottky group of orientation preserving isometries of $\mathbb{H}^{n+1}$ with $n+1$ is even and assume that $\delta$, the Hausdorff dimension of the limit set, is larger or equal to $n / 2$. Then the Krein function for $X=\Gamma \backslash \mathbb{H}^{n+1}$ satisfies the Breit-Wigner approximation: for $\sigma>0$ fixed and all $z \in[T-\sigma, T+\sigma]$ with $T$ large,

$$
\partial_{z} \xi(z)=\chi(X) P_{n}(z)+\frac{1}{\pi} \sum_{\rho \in \mathcal{R}_{\sigma+1}} \frac{\operatorname{Im}(\rho)}{|z-\rho|^{2}}+\mathcal{O}\left(T^{\delta}\right)
$$

where $\mathcal{R}_{\sigma+1}$ is the set of resonances in the disc of radius $\sigma+1$ centered at $T$, and $P_{n}(z)$ is an odd polynomial of degree $n, \chi(X)$ the Euler characteristic of $X$. Moreover the following asymptotics holds

$$
\xi(z)=\chi(X) P_{n+1}(z)+R(z), \quad|R(z)|=\mathcal{O}\left(z^{\delta}\right), \quad\left|\int_{0}^{T} R(z) d z\right|=\mathcal{O}\left(T^{\delta}\right)
$$

where $P_{n+1}(z)$ is an even polynomial of degree $n+1$.

3.3. The case of surfaces. A convex co-compact surface $M=\Gamma \backslash \mathbb{H}^{2}$ decomposes into a compact hyperbolic surface with totally geodesic boundary (the convex core $N$ ) and a collection of $n_{f}$ ends which are funnels $F_{j}$ isometric to

$$
[0, \infty)_{t} \times\left(\mathbb{R} / \ell_{j} \mathbb{Z}\right)_{\theta} \text { with metric } d t^{2}+\cosh (t)^{2} d \theta^{2}
$$

This can be seen as half of the hyperbolic cylinder $\langle\gamma\rangle \backslash \mathbb{H}^{2}$ where $\langle\gamma\rangle$ is the cyclic elementary group generated by one single hyperbolic isometry with translation length $\ell_{j}$. Guillopé-Zworski [GuZw] studied the spectral shift function $s(z)^{1}$, related to the pair of operators $\left(\Delta_{M}, \Delta_{F}\right)$ where $\Delta_{F}:=\bigoplus_{j=1}^{n_{f}} \Delta_{F_{j}}$ where $\Delta_{F_{j}}$ is the Laplacian on $F_{j}$ with Dirichlet condition at the boundary of $F_{j}$ (given by $t=0$ in the coordinate above). They define $s(z)=\frac{i}{2 \pi} \log \operatorname{det} \mathcal{S}(1 / 2+i z)$ for $z \in \mathbb{R}$, where $\mathcal{S}(s)$ is the relative scattering operator, and they show that $s^{\prime}(z)$ is meromorphic in $z \in \mathbb{C}$ with poles and zeros given essentially by resonances of the Laplacians. We do not give details and refer the interested reader to [GuZw]. In fact, this function $s(z)$ is given by

$$
s^{\prime}(z)=\xi^{\prime}(z)-\xi_{F}^{\prime}(z)
$$

where $\xi$ is the spectral function of (3.4) for $M$ and $\xi_{F}=\sum_{j} \xi_{F_{j}}(z)$ where $\xi_{F_{j}}$ is defined the same way as (3.4) on the funnel $F_{j}$ (and for the spectral measure of $\Delta_{F_{j}}$ ); see the

\footnotetext{
${ }^{1} \mathrm{In}[\mathrm{GuZw}]$, it was denoted $\sigma(z)$ instead of $s(z)$
} 
proof of Proposition 4.4 in [BoJuPe]. It is computed by [BoJuPe, (2.10)] that there is a constant $c$ such that

$$
e^{2 \pi i \xi_{F_{j}}(z)+c}=\frac{Z_{F_{j}}(1 / 2+i z)}{Z_{F_{j}}(1 / 2-i z)}
$$

where $Z_{F_{j}}(s):=e^{-s \ell_{j} / 4} \prod_{k \geq 0}\left(1-e^{-(s+2 k+1) \ell_{j}}\right)^{2}$ is the Selberg zeta function for the funnel $F_{j}$. This function converges absolutely for $\operatorname{Re}(s)>0$ and in particular on the line $1 / 2+i \mathbb{R}$. From this convergence, we directly see that $\xi_{F_{j}}^{\prime}(z)=\mathcal{O}(1)$ and we also deduce from Lemma 3.3 (as we did before for $Z_{\Gamma}$ ) that $\xi_{F_{j}}(z)=\mathcal{O}(1)$ for $z \in \mathbb{R}$. Thus by combining with Theorem 5 and the Gauss-Bonnet formula $\operatorname{Vol}(K)=-2 \pi \chi(M)=$ $2 \pi\left(2 g-2+n_{f}\right)$ (where $g$ is the genus), we obtain

Theorem 6. The scattering phase defined by Guillopé-Zworski [GuZw] for hyperbolic convex co-compact surfaces $M=\Gamma \backslash \mathbb{H}^{2}$ satisfies the asymptotics as $z \rightarrow+\infty$

$$
s(z)=\frac{1}{4 \pi} \operatorname{Vol}(K) z^{2}+R(z), \quad|R(z)|=\mathcal{O}\left(z^{\delta}\right), \quad\left|\int_{0}^{z} R(t) d t\right|=O\left(z^{\delta}\right)
$$

where $K$ is the convex core of $M, \delta$ is the Hausdorff dimension of the limit set of $\Gamma$. Moreover the Breit-Wigner formula holds: for all $z \in[T-\sigma, T+\sigma]$ with $T$ large and $\sigma$ fixed

$$
\partial_{z} s(z)=\frac{1}{4 \pi} \operatorname{Vol}(K) z^{2}+\frac{1}{\pi} \sum_{\rho \in \mathcal{R}_{\sigma+1}} \frac{\operatorname{Im}(\rho)}{|z-\rho|^{2}}+\mathcal{O}\left(z^{\delta}\right)
$$

In $[\mathrm{GuZw}]$, an asymptotics with a remainder $\mathcal{O}(z)$ using the method of Melrose [Me88] was proved. Our remainder estimate is thus always better than [GuZw] since $\delta<1$.

3.4. Lower bounds for the remainder. For arithmetic hyperbolic surfaces, one can use the argument of Jakobson-Naud [JaNa] to get a polynomial Omega lower bound on the argument of Selberg zeta function on the line $\operatorname{Re}(s)=1 / 2$ :

Proposition 3.6. [Jakobson-Naud] Let $\Gamma$ be a convex co-compact subgroup of isometries of $\mathbb{H}^{2}$, which is an infinite index subgroup of an arithmetic group $\Gamma_{0}$ derived from a quaternion algebra. Assume that the Hausdorff dimension $\delta$ of the limit set satisfies $\delta>3 / 4$, then for all $\varepsilon>0$, the argument $\operatorname{Arg}\left(Z_{\Gamma}(1 / 2+i z)\right)$ of Selberg zeta function for $z \in \mathbb{R}$ is not an $\mathcal{O}\left(z^{2 \delta-3 / 2-\varepsilon}\right)$.

Proof. Let $Z_{\Gamma}(\lambda)$ be the Selberg zeta function of $\Gamma$. In [JaNa, Proposition 3.4], it is shown that there exist some constants $A>0, C>0$ such that for all $T>0$ large and $\alpha=2 \log (T)-A$, then

$$
\int_{T}^{3 T}\left|S_{\alpha, t}\right|^{2} d t \geq \frac{C T^{4 \delta-2}}{(\log T)^{2}}
$$


where $S_{\alpha, t}$ is defined for $\alpha>0, t>0$ as follows: let $\varphi_{0} \in C_{0}^{\infty}((-1,1))$ non-negative equal to 1 in $[-1 / 2,1 / 2]$, then set $\varphi_{t, \alpha}(x)=e^{-i t x} \varphi_{0}(x-\alpha)$ and

$$
\begin{aligned}
S_{\alpha, t} & :=\sum_{k \geq 1} \sum_{\gamma} \frac{\ell(\gamma)}{2 \sinh (k \ell(\gamma) / 2)} e^{-i t k \ell(\gamma)} \varphi_{0}(k \ell(\gamma)-\alpha) \\
& =\frac{1}{\pi}\left\langle\partial_{z} \operatorname{Arg}\left(Z_{\Gamma}\left(\frac{1}{2}+i z\right)\right), \widehat{\varphi_{t, \alpha}}(z)\right\rangle=-\frac{1}{\pi}\left\langle\operatorname{Arg}\left(Z_{\Gamma}\left(\frac{1}{2}+i z\right)\right), \partial_{z} \widehat{\varphi_{t, \alpha}}(z)\right\rangle .
\end{aligned}
$$

Now, assume that $\operatorname{Arg}\left(Z_{\Gamma}\left(\frac{1}{2}+i z\right)\right)=\mathcal{O}\left(z^{2 \delta-3 / 2-\varepsilon}\right)$ for some $\varepsilon>0$, then since $\left|\partial_{z} \widehat{\varphi_{t, \alpha}}(z)\right|=$ $\mathcal{O}\left((1+|t+z|)^{-N}\right)$ for all $N \in \mathbb{N}$, we get for $t>0$ large

$$
\left|S_{\alpha, t}\right| \leq C t^{2 \delta-3 / 2-\varepsilon}
$$

where $C$ does not depend on $\alpha$. This estimate contradicts (3.12) for $T$ large enough, this ends the proof.

Note that such groups exist, see [JaNa, Section 4]. By (3.11) and (3.5), we then deduce the

Corollary 3.7. If $\Gamma$ is a convex co-compact infinite index subgroup of an arithmetic group $\Gamma_{0}$ derived from a quaternion algebra, and if the Hausdorff dimension $\delta$ of the limit set of $\Gamma$ satisfies $\delta>3 / 4$, then for all $\varepsilon>0$ the remainder $R(z)$ in the scattering phase asymptotics of Theorem 6 is not an $\mathcal{O}\left(z^{2 \delta-3 / 2-\varepsilon}\right)$.

In general, without the arithmetic assumption, the argument of Jakobson-Naud [JaNa] shows that for all $\varepsilon>0$, if $\delta>1 / 2$ then the remainder $R(z)$ is not an $\mathcal{O}\left((\log z)^{\frac{\delta-1 / 2}{\delta}-\varepsilon}\right)$.

Acknowledgements. C.G. is supported by ANR grant ANR-09-JCJC-0099-01. S.D. was partially supported by NSF grant DMS-0654436. We thank Johannes Sjöstrand and Maciej Zworski for answering our questions on the counting functions for resonances, as well as Frédéric Naud and Vesselin Petkov for useful discussions and references.

\section{REFERENCES}

[Bé] P. Bérard, On the wave equation on a compact manifold without conjugate points, Math. Z. 155(1977), no. 3, 249-276.

[BiKr] M.S. Birman and M.G. Krein, On the theory of wave operators and scattering operators, Dokl. Akad. Nauk SSSR 144(1962), 475-478; English translation in Soviet. Math. Dokl 3(1962).

[Bo] D. Borthwick, Upper and lower bounds on resonances for manifolds hyperbolic near infinity, Comm. PDE 33(2008), 1507-1539.

[BoJuPe] D. Borthwick, C. Judge, and P. Perry, Selberg's zeta function and the spectral geometry of geometrically finite hyperbolic surfaces, Comment. Math. Helv. 80(2005), no. 3, 483-515.

[BoRu] R. Bowen and D. Ruelle, The ergodic theory of Axiom A flows, Invent. Math. 29(1975), $181-202$.

[BrPe] V. Bruneau and V. Petkov, Meromorphic continuation of the spectral shift function, Duke Math. J, 116(2003), no. 3, 389-430. 
[Bu] V.S. Buslaev, Local spectral asymptotic behavior of the Green's function in exterior problems for the Schrödinger operator, (Russian. English summary) Collection of articles dedicated to the memory of Academician V. I. Smirnov. Vestnik Leningrad. Univ. No. 1 Mat. Meh. Astronom. Vyp. 1 (1975), 55-60, 189.

[Chr] T. Christiansen, Spectral asymptotics for compactly supported perturbations of the Laplacian on $\mathbb{R}^{n}$, Comm. PDE 23(1998), no. 5-6, 933-948.

[Di] M. Dimassi, Spectral shift function and resonances for slowly varying perturbations of periodic Schrdinger operators. J. Funct. Anal. 225 (2005), no. 1, 193228.

[DiSj] M. Dimassi and J. Sjöstrand, Spectral asymptotics in the semi-classical limit, Cambridge University Press, 1999.

[DyGu] S. Dyatlov and C. Guillarmou, Microlocal limits of plane waves and Eisenstein functions, preprint, arXiv:1204.1305.

[Gr] C.R. Graham, Volume and area renormalizations for conformally compact Einstein metrics, Rend. Circ. Mat. Palermo, Ser.II, Suppl. 63 (2000), 31-42.

[Gu] C. Guillarmou, Generalized Krein formula, determinants and Selberg zeta function in even dimension, Amer. J. Math. 131(2009), no 5.

[GuLiZw] L. Guillopé, K. Lin, and M. Zworski, The Selberg zeta function for convex co-compact Schottky groups, Comm. Math. Phys. 245(2004), no. 1, 149-176.

[GuZw] L. Guillopé and M. Zworski, Scattering asymptotics for Riemann surfaces, Ann. Math. 145(1997), 597-660.

[HeRo] B. Helffer and D. Robert, Calcul fonctionnel par la transformée de Mellin et opérateurs admissibles, J. Func. Anal. 53(1983), no 3, 246-268.

[HöIII] L. Hörmander, The Analysis of Linear Partial Differential Operators III. Pseudo-differential Operators, Springer, 1985.

[Iv] V. Ivrii, The second term of the spectral asymptotics for a LaplaceBeltrami operator on manifolds with boundary, Funktsional. Anal. i Prilozhen 14(1980), no. 2, 25-34.

[JaNa] D. Jakobson, F. Naud, Lower bounds for resonances of infinite area Riemann surfaces. Analysis and PDE 3 (2010), no 2, 207-225.

[JeKa] A. Jensen and T. Kato, Asymptotic behavior of the scattering phase for exterior domains, Comm. PDE 3(1978), no. 12, 1165-1195.

[MaRa1] A. Majda and J. Ralston, An analogue of Weyl's theorem for unbounded domains. I, Duke Math. J. 45(1978), no. 1, 183-196.

[MaRa2] A. Majda and J. Ralston, An analogue of Weyl's theorem for unbounded domains. II, Duke Math. J. 45(1978), no. 3, 513-536.

[MaMe] R.R. Mazzeo and R.B. Melrose, Meromorphic extension of the resolvent on complete spaces with asymptotically constant negative curvature, J. Funct. Anal. 75(1987), no. 2, 260-310.

[Me83] R.B. Melrose, Polynomial bound on the number of scattering poles, J. Funct. Anal. 53(1983), no. 3, 287-303.

[Me88] R.B. Melrose, Weyl asymptotics for the phase in obstacle scattering, Comm. PDE 13(1988), no. 11, 1431-1439.

[Pa] S.J. Patterson, The limit set of a Fuchsian group, Acta Math. 136(1976), no. 3-4, 241-273.

[PaPe] S. Patterson and P. Perry, The divisor of Selberg's zeta function for Kleinian groups. Appendix A by Charles Epstein, Duke Math. J. 106(2001), 321-391.

[PePo] V. Petkov and G. Popov, Asymptotic behaviour of the scattering phase for non-trapping obstacles, Ann. Inst. Fourier 32(1982), 111-149. 
[PeZw] V. Petkov and M. Zworski, Breit-Wigner approximation and distribution of resonances, Comm. Math. Phys. 204(1999), 329-351.

[Ro] D. Robert, Asymptotique de la phase de diffusion à haute énergie pour des perturbations du second ordre du laplacien, Ann. Sci. École Norm. Sup. (4) 25(1992), no. 4, 107-134.

[Sj90] J. Sjöstrand, Geometric bounds on the density of resonances for semiclassical problems, Duke Math. J. 60(1990), no. 1, 1-57.

[Sj97] J. Sjöstrand, A trace formula and review of some estimates for resonances, in Microlocal analysis and spectral theory (Lucca 1997), 377-437, NATA Adv. Sci. Inst. Ser. C Math. Phys Sci., 490, Dordrecht, Kluwer Acad. Publ. 1997.

[SjZw91] J. Sjöstrand and M. Zworski, Complex scaling and the distribution of resonances, J. Amer. Math. Soc. 4(1991), 729-769.

[SjZw94] J. Sjöstrand and M. Zworski, Lower bounds on the number of scattering poles, II, J. Funct. Anal. 123(1994), 336-367.

[SjZw07] J. Sjöstrand and M. Zworski, Fractal upper bounds on the density of semiclassical resonances, Duke Math. J. 137(2007), no. 3, 381-459.

[Su] D. Sullivan, The density at infinity of a discrete group of hyperbolic motions, Publ. Math. de l'IHES, 50(1979), 171-202.

[Ti] E.C. Titchmarsh, The theory of the Riemann zeta function, Second edition. Clarendon Press, Oxford, 1986.

[Vo] G. Vodev, Sharp polynomial bound on the number of scattering poles for metric perturbations of the Laplacian in $\mathbb{R}^{n}$, Math. Ann. 291(1991), 39-49.

[Yo] L.S. Young, Some large deviation results for dynamical systems, Trans. Amer. Math. Soc. 318(1990), no. 2, 325-343.

[Zw97] M. Zworski, Poisson formulae for resonances, Séminaire sur les Équations aux Dérivées Partielles, 1996-1997, Exp. No. XIII, 14 pp.

[Zw] M. Zworski, Semiclassical analysis, to appear in Graduate Studies in Mathematics, AMS, 2012.

E-mail address: dyatlov@math.berkeley.edu

Department of Mathematics, Evans Hall, University of California, Berkeley, CA 94720, USA

E-mail address: cguillar@dma.ens.fr

DMA, U.M.R. 8553 CNRS, École Normale Superieure, 45 Rue D'Ulm, 75230 Paris CEDex 05, France 